\title{
FATORES PROGNÓSTICOS EM PACIENTES COM CARCINOMA DAS CÉLULAS DE HÜRTHLE
}

\author{
PROGNOSTIC FACTORS IN PATIENTS WITH HÜRTHLE CELL CARCINOMA
}

\author{
Gabriel Manfro' ${ }^{1}$ Fernando Luiz Dias, TCBC-RJ²; Roberto Araujo Lima, TCBC-RJ ${ }^{3}$; \\ Mauro Marques Barbosa, TCBC-RJ ${ }^{3}$; José Roberto Netto Soares ${ }^{3}$; Marilena Figueira Nascimento ${ }^{4}$
}

\begin{abstract}
RESUMO: Objetivo: Analisar fatores prognósticos na sobrevida livre de doença e específica dos pacientes com carcinomas de células de Hürthle. Método: Estudo retrospectivo de 28 pacientes tratados na Seção de Cirurgia de Cabeça e Pescoço do HCI - INCa, objetivando coletar dados demográficos, clínicos e histopatológicos e relacioná-los com a sobrevida livre de doença e sobrevida global específica. O estadiamento foi realizado conforme a AJCC de 2002. Para análise estatística foi utilizado o programa Epi info 2002, considerando-se significativo estatisticamente o valor de $\mathrm{p}<0,05$. A análise da curva de sobrevida foi realizada utilizando-se o método de Kaplan-Meyer. O tempo médio de seguimento foi de 69,3 meses (10 - 230 meses). Resultados: Foram avaliados 28 pacientes, com idade média de 50,8 anos. As variáveis que apresentaram impacto prognóstico quanto a recidiva foram o estágio mais avançado da doença $(p=0,03)$, a presença de metástase a distância $(p=0,03$ e principalmente o padrão histológico de invasão maciça $(p=0,0027)$. Quando relacionado as variáveis estudadas com a mortalidade, o padrão de invasão histológica maciça ( $p=$ $0,02)$, o maior tamanho do tumor $(p=0,013)$ e principalmente a presença de metástases a distância $(p=0,0056)$ apresentaram relação com significância estatística. A sobrevida livre de doença foi de $72 \%$ e $55 \%$ e a sobrevida global de $87 \%$ e $77 \%$ em 5 e 10 anos respectivamente. Conclusão: A presença de metástase à distância e o padrão histológico de invasão maciça apresentaram relação estatisticamente significativa com a sobrevida livre de doença e específica (Rev. Col. Bras. Cir. 2006; 33(2): 84-90).
\end{abstract}

Descritores: Células oxífilas; Glândula tireóide; Carcinoma; Prognóstico; Neoplasia de glândula tireóide; Adenoma oxífilo; Sobrevivência livre de doença.

\section{INTRODUÇÃO}

O carcinoma das células de Hürthle $(\mathrm{CCH})$ é um tumor raro da tireóide representando menos que $5 \%$ dos tumores bem diferenciados desta glândula ${ }^{1}$. As células dos tumores de Hürthle foram primeiramente descritas por Askanazy ${ }^{2}$, e têm como características morfológicas um grande tamanho, podendo ter o formato oval ou poligonal, limites bem definidos, citoplasma granular volumoso, devido à presença de mitocôndrias, núcleos grandes e nucléolos múltiplos, sendo um deles proeminente. Estas células contêm altos níveis de enzimas oxidativas. As células de Hürthle podem ser encontradas em várias doenças da tireóide como a tireoidite linfocítica, doença de Graves e bócio nodular, não podendo ser considerada específica de nenhuma delas ${ }^{2}$.

Nem todos os nódulos que apresentam células de Hürthle são neoplásicos, a maioria das células está presente em nódulos de adenomas foliculares, bócios e tireoidites. Quando estas células correspondem a mais de $75 \%$ da celularidade do nódulo, este é denominado neoplasia ${ }^{2}$.

O carcinoma de células de Hürthle $(\mathrm{CCH})$ é considerado pela Organização Mundial da Saúde desde 1963, uma variante do carcinoma folicular da glândula tireóide. No entanto, o CCH apresenta expressões oncogênicas diferentes quando comparado ao carcinoma folicular, sendo em alguns relatos considerado uma doença distinta ${ }^{1}$. As evidências que mostram que o $\mathrm{CCH}$ tem origem folicular são baseadas em alguns estudos histológicos evidenciando numerosas formas de transição entre uma célula folicular e uma célula de Hürthle ${ }^{2}$.

O comportamento biológico do $\mathrm{CCH}$ ainda é bastante discutido, fazendo com que haja a necessidade de uma melhor definição de quais fatores demográficos, clínicos e histológicos apresentam impacto no prognóstico dos pacientes.

Resolvemos então analisar os fatores de impacto prognóstico na sobrevida livre de doença e sobrevida específica dos pacientes com carcinomas de células de Hürthle operados pela Seção de Cirurgia de Cabeça e Pescoço do Instituto Nacional de Câncer - INCa, Rio de Janeiro - Brasil, entre 1983 a 2002.

\section{MÉTODO}

Foi realizado um estudo retrospectivo com análise de prontuários dos pacientes matriculados na seção de Cirur-

\footnotetext{
1. Residente R3 da Seção de Cirurgia de Cabeça e Pescoço do Instituto Nacional de Câncer - INCA - Rio de Janeiro - RJ.

2. Chefe da Seção de Cirurgia de Cabeça e Pescoço do Instituto Nacional de Câncer - INCA - Rio de Janeiro - RJ.

3. Titulares da Seção de Cirurgia de Cabeça e Pescoço do Instituto Nacional de Câncer - INCA - Rio de Janeiro -RJ.

4. Titular da Divisão de Anatomia Patológica do Instituto Nacional de Câncer - INCA - Rio de Janeiro - RJ.

Recebido em 14/10/2005

Aceito para publicação em 16/12/2005

Conflito de interesses : nenhum

Fonte de financiamento: nenhuma

Trabalho realizado na Seção de Cirurgia de Cabeça e Pescoço do Instituto Nacional de Câncer - INCA - Rio de Janeiro.
} 
Tabela 1 - Estadiamento dos carcinoma bem diferenciados da glândula tireóide conforme American Joint Committe on Câncer (AJCC) de 2002.

\begin{tabular}{ll}
\hline Tx & Tumor primário não pode ser avaliado. \\
T0 & Sem evidência de tumor primário. \\
T1 & Tumor com $2 \mathrm{~cm}$ ou menos na sua maior dimensão, limitado à tireóide. \\
T2 & Tumor com mais de $2 \mathrm{~cm}$, mas não mais que $4 \mathrm{~cm}$ na sua maior dimensão, limitado à tireóide. \\
T3 & $\begin{array}{l}\text { Tumor com mais de } 4 \mathrm{~cm} \text { na sua maior dimensão, limitado à tireóide, ou qualquer tumor com mínima extensão extra- } \\
\text { tireoideana. }\end{array}$ \\
T4a & $\begin{array}{l}\text { Tumor de qualquer tamanho que se estende além da cápsula da tireóide invade tecidos moles subcutâneos, laringe, } \\
\text { traquéia, esôfago ou nervo laríngeo recorrente. }\end{array}$ \\
T4b & Tumor invade fáscia pré-vertebral ou engloba a artéria carótida ou os vasos mediastinais. \\
Nx & Linfonodos regionais não podem ser avaliados. \\
N0 & Ausência de metástases em linfonodos regionais. \\
N1 & Metástases em linfonodos regionais. \\
N1a & Metástases para o nível VI. \\
N1b & Metástases em linfonodos cervicais uni, bi, contralaterais ou mediastinais superiores. \\
Mx & Metástases à distância não podem ser avaliadas. \\
M0 & Ausência de metástases à distância. \\
M1 & Metástases à distância.
\end{tabular}

\begin{tabular}{|c|c|c|c|c|}
\hline \multicolumn{5}{|c|}{$<45$ anos } \\
\hline & EI & Qualquer T & Qualquer N & M0 \\
\hline & EII & Qualquer T & Qualquer N & M1 \\
\hline \multicolumn{5}{|c|}{$\geq 45$ anos } \\
\hline & EI & $\mathrm{T} 1$ & No & M0 \\
\hline & EII & $\mathrm{T} 2$ & No & M0 \\
\hline & E III & $\mathrm{T} 3$ & N0, N1a & M0 \\
\hline & & $\mathrm{T} 1, \mathrm{~T} 2, \mathrm{~T} 3$ & N1a & M0 \\
\hline & EIVa & $\mathrm{T} 4 \mathrm{a}$ & N0, N1a, N1b & M0 \\
\hline & & $\mathrm{T} 1, \mathrm{~T} 2, \mathrm{~T} 3, \mathrm{~T} 4 \mathrm{a}$ & $\mathrm{N} 1 \mathrm{~b}$ & M0 \\
\hline & $\mathrm{EIVb}$ & $\mathrm{T} 4 \mathrm{~b}$ & Qualquer N & M0 \\
\hline & EIVc & Qualquer T & Qualquer N & M1 \\
\hline
\end{tabular}

gia de Cabeça e Pescoço do Instituto Nacional de Câncer/ INCA, operados por Carcinoma de Células de Hürthle.

De um total de 36 pacientes, oito foram excluídos por não apresentarem informações completas nos prontuários. Vinte e oito pacientes foram estudados quanto a idade, sexo, tamanho do tumor $(\mathrm{T})$, metástase linfática $(\mathrm{N})$, metástase a distância (M), tipo de cirurgia realizada e padrão histológico de invasão, sendo estas variáveis relacionados com a recidiva da doença e incidência de óbito pela doença.

Conforme a idade, os pacientes foram divididos em dois grupos. O primeiro com idade menor ou igual aos 40 anos e o segundo com idade maior que 40 anos.

A divisão dos pacientes de acordo com o tamanho do tumor, presença de metástase linfática e à distância, foi realizada baseada na classificação da American Joint Committe on Cancer - AJCC de 2002 (Tabela 1).

Quanto ao procedimento cirúrgico realizado, o primeiro grupo foi composto por aqueles que foram submetidos à tireoidectomia menor que a tireoidectomia total, e o segundo por pacientes submetidos a tireoidectomia total associada ou não a linfadenectomia.

De acordo com a análise histológica os tumores foram divididos em minimamente invasivos (Mil) e maciçamente invasivos (MaI). Foram classificados como minimamente invasivos quando havia apenas um foco de invasão vascular intra ou extra capsular ou um foco completo de invasão capsular. Os tumores maciçamente invasivos eram caracterizados por mais de um foco de invasão vascular ou de invasão capsular ${ }^{1}$. Todos os casos foram reanalisados pelo mesmo patologista (MN). O tempo médio de seguimento foi de 69,3 meses (10 - 230 meses). Para análise estatística foi utilizado o programa Epi info 2002, considerando-se significativo estatisticamente o valor de $\mathrm{p}<0,05$. A análise da curva de sobrevida foi realizada utilizando-se o método de Kaplan-Meyer.

\section{RESULTADOS}

Estudou-se um total de 28 pacientes no período entre outubro de 1981 e setembro de 2002 . Vinte pacientes $(71,4 \%)$ eram do sexo feminino e oito $(28,6 \%)$ do sexo masculino, com uma média de idade de 50,8 anos ( $15-76$ anos) e mediana de 51 anos. Dezessete pacientes $(71,4 \%)$ tinha idade maior que 40 anos e apenas oito $(28,6 \%)$ apresentavam idade menor ou igual a 40 anos na época do tratamento.

Todos os pacientes foram submetidos ao tratamento operatório, sendo que em seis $(21,4 \%)$ foi realizada lobectomia 
Tabela 2 - Análise univariada comparando variáveis clínicas, histopatológicas e cirúrgicas com a sobrevida livre de doença dos pacientes com carcinoma das células de Hürthle.

\begin{tabular}{|c|c|c|c|}
\hline Fator Prognóstico & $\mathbf{N}^{\circ}$ pacientes & $\begin{array}{c}\text { Sobrevida livre de doença } \\
\text { em } 5 \text { / } 10 \text { anos }\end{array}$ & $p$ \\
\hline \multirow[t]{2}{*}{ Sexo } & Masculino: 8 & $66 \% / 66 \%$ & \\
\hline & Feminino: 20 & $72 \% / 54 \%$ & 0,62 \\
\hline \multirow[t]{2}{*}{ Idade } & $>40$ anos: 20 & $65 \% / 48 \%$ & \\
\hline & $\leq 40$ anos: 8 & $83 \% / 67 \%$ & 0,82 \\
\hline \multirow[t]{3}{*}{$\mathrm{pT}$} & T2: 17 & $70 \% / 70 \%$ & \\
\hline & T3: 6 & $75 \% / 75 \%$ & 0,14 \\
\hline & $\mathrm{T} 4: 5$ & $75 \% / 23 \%$ & \\
\hline \multirow[t]{2}{*}{$\mathrm{N}$} & N0: 24 & $88 \% / 65 \%$ & \\
\hline & $\mathrm{N}+: 4$ & $23 \% / 23 \%$ & 0,06 \\
\hline \multirow[t]{2}{*}{ M } & M0: 22 & $84 \% / 72 \%$ & \\
\hline & M1: 6 & $41 \% / 20 \%$ & 0,033 \\
\hline \multirow[t]{4}{*}{ Estágio } & $\mathrm{I}: 8$ & $100 \% / 100 \%$ & \\
\hline & II: 11 & $76 \% / 57 \%$ & \\
\hline & III: 5 & $50 \% / 50 \%$ & \\
\hline & IV: 4 & $66 \% / 0 \%$ & 0,03 \\
\hline \multirow[t]{2}{*}{ Cirurgia } & Total: 22 & $64 \% / 46 \%$ & \\
\hline & Parcial: 6 & $100 \% / 100 \%$ & 0,28 \\
\hline \multirow[t]{2}{*}{ Padrão Histológico } & MiI: 14 & $100 \% / 100 \%$ & \\
\hline & MaI: 14 & $49 \% / 15 \%$ & 0,0027 \\
\hline
\end{tabular}

com istmectomia. Nos outros $22(78,6 \%)$ pacientes foi realizada tireoidectomia total. O esvaziamento cervical póstero-lateral e recorrencial (Níveis II-VI) foi realizado em três casos $(10,7 \%)$.

O tamanho médio do tumor foi de $3,23 \mathrm{~cm}$. Dezessete pacientes $(60,8 \%)$ apresentavam um tumor primário medindo entre 2 e $4 \mathrm{~cm}$ sendo estadiados como pT2, $6(21,4 \%)$ pacientes foram classificados como pT3 e em apenas $5(17,8 \%)$ pacientes havia extravasamento da cápsula da glândula tireóide no estudo anátomo-patológico (pT4).

As metástases para linfonodos cervicais foram diagnosticadas em apenas quatro (14,3\%) pacientes, e em três deles foi realizado esvaziamento cervical póstero-lateral e recorrencial ( Níveis linfáticos cervicais II - VI). Destes quatro pacientes com metástase cervical dois apresentaram recidiva à distância nos ossos, um teve recidiva regional e foi submetido a esvaziamento cervical. Somente um paciente não apresentou recidiva.

Em $15(53,6 \%)$ casos o tratamento foi complementado com iodo radioativo com a dose média de $200 \mathrm{mCi}(93-450)$. Destes 15 pacientes, apenas um $(6,6 \%)$ havia sido submetido à lobectomia com istmectomia, dois $(13,4 \%)$ foram tratados com tireoidectomia associada ao esvaziamento cervical póstero-lateral e os outros $12(80 \%)$ pacientes tiveram como primeiro tratamento a tireoidectomia total. Não houve diferença significativa entre o grupo de pacientes que recebeu radioiodoterapia com o grupo que não recebeu tratamento complementar ao serem analisados a recidiva e a mortalidade, $\operatorname{com} p=0,82$ e 0,17 , respectivamente.

O tipo de operação (total versus lobectomia com istmectomia) não apresentou influência significativamente estatística quando comparado com a recidiva $(p=0,28)$ e a mortalidade $(p=0,83)$. Não houve recidiva da doença no gru- po submetido a tireoidectomia parcial. Os sete $(25 \%)$ pacientes que apresentaram recidiva foram tratados com tireoidectomia total que em dois pacientes foi associada ao esvaziamento cervical.

Dos seis $(21,4 \%)$ pacientes submetidos à lobectomia com istmectomia, nenhum evoluiu para óbito. Os três $(10,7 \%)$ pacientes que morreram, haviam sido submetidos a tireoidectomia total e tiveram a causa do óbito relacionada com a doença tireoideana.

Sete $(25 \%)$ pacientes apresentaram recidiva da doença, em três $(42,8 \%)$ casos regional e em quatro $(57,2 \%)$ casos recidiva à distância, sendo em todos estes casos metástase óssea, três desses pacientes evoluíram à óbito.

Em seis $(21,4 \%)$ pacientes foi evidenciada metástase à distância durante alguma fase do tratamento, sendo em 5 $(83,4 \%)$ casos a metástase localizava-se nos ossos e em um $(16,6 \%)$ caso ocorreu no pulmão.

A metade dos pacientes apresentou ao estudo histológico um padrão de invasão mínimo (MiI). Os outros 14 (50\%) pacientes apresentaram invasão maciça (MaI).

A sobrevida média global foi de 69,29 meses e a tempo médio livre de doença foi de 49,2 meses. A sobrevida livre de doença foi de $72 \%$ e $55 \%$ (Figura 1) e a sobrevida global de $87 \%$ e $77 \%$ (Figura 2) em 5 e 10 anos respectivamente.

Os fatores de impacto mais importantes para a recidiva foram: o estágio mais avançado da doença $(p=0,03)$, a presença de metástase à distância $(p=0,03)$ e principalmente o padrão histológico de invasão maciça $(p=0,0027)$ (Tabela 2).

Quando analisada a mortalidade, os fatores de maior impacto foram: o padrão histológico de invasão maciça ( $p=$ $0,02)$, o maior tamanho do tumor $(p=0,013)$ e principalmente a 


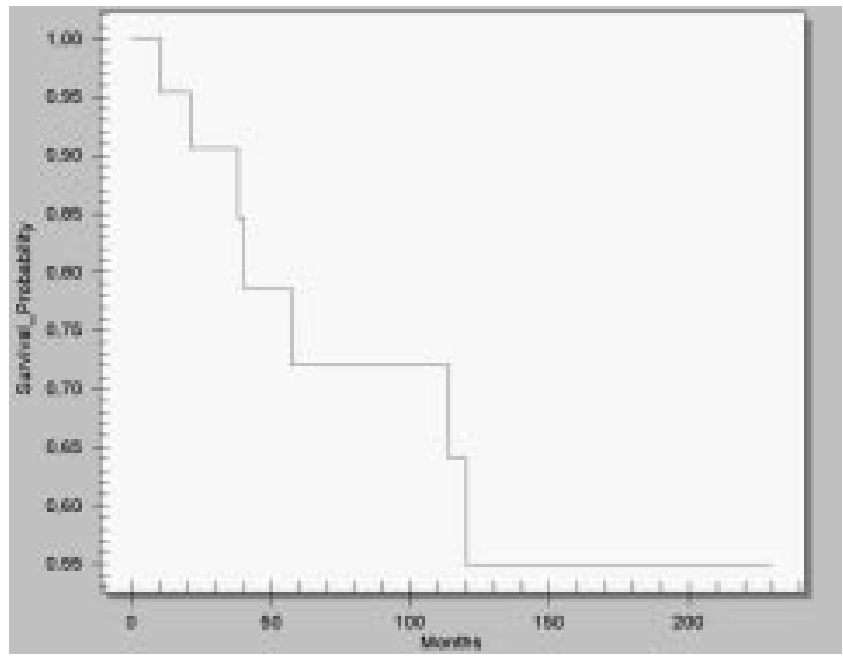

Figura 1 - Curva de sobrevida livre de doença dos pacientes com Carcinoma de células de Hürthle.

presença de metástase à distância $(p=0,0056)$ (Tabela 3). Quando avaliamos a influência de outras variáveis sobre a incidência de metástases à distância, a presença de metástase linfática apresentou significância estatística com a presença de metástases à distância ( $p=0,003$ ) (Tabela 4). O padrão histológico de invasão maciça apresentou relação com o maior tamanho do tumor $(p=0,05)$ e a presença de metástases à distância $(p=0,02)$ (Tabela 5).

\section{DISCUSSÃo}

Muitos autores consideram o $\mathrm{CCH}$ mais agressivo que os carcinomas bem diferenciados da tireóide ${ }^{2,3}$, ao contrário do

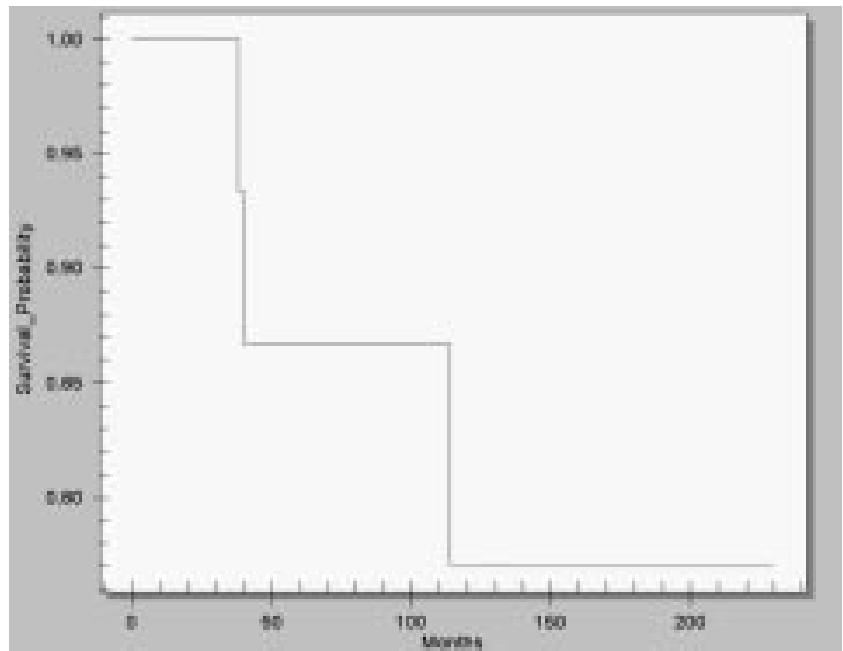

Figura 2 - Curva de sobrevida específica dos pacientes com Carcinoma de células de Hürthle.

que foi descrito por Khafif em 1999, onde o CCH teve evolução mais favorável que o carcinoma folicular ${ }^{4}$. Sanz em 1999 descreveu um comportamento biológico e tratamento semelhante entre o $\mathrm{CCH}$ e os carcinomas de tireóide bem diferenciados ${ }^{5}$.

A diferenciação entre o adenoma e o carcinoma de células de Hürthle é difícil de ser avaliada. Não há marcador imuno-histoquímico específico para tal diferenciação ${ }^{2}$. O tamanho, a atipia nuclear, a multinucleação, o pleomorfismo celular, a presença de mitoses, o grau de aneuploidia e o padrão histológico não são preditivos de malignidade. Os padrões de malignidade são as invasões: vascular e capsular destrutiva, dados estes impossibilitados de serem caracterizados em material de punção aspirativa com agulha fina (PAAF).

Tabela 3 - Análise univariada comparando variáveis clínicas, histopatológicas e cirúrgicas com a sobrevida específica dos pacientes com Carcinoma das células de Hürthle.

\begin{tabular}{|c|c|c|c|}
\hline Fator Prognóstico & $\mathbf{N}^{\circ}$ pacientes & $\begin{array}{c}\text { Sobrevida livre de doença } \\
\text { em } 5 \text { / } 10 \text { anos }\end{array}$ & $p$ \\
\hline \multirow[t]{2}{*}{ Sexo } & Masculino: 8 & $66 \% / 66 \%$ & \\
\hline & Feminino: 20 & $72 \% / 54 \%$ & 0,62 \\
\hline \multirow[t]{2}{*}{ Idade } & $>40$ anos: 20 & $89 \% / 89 \%$ & \\
\hline & $\leq 40$ anos: 8 & $83 \% / 67 \%$ & 0,68 \\
\hline \multirow[t]{3}{*}{ pT } & $\mathrm{T} 2: 17$ & $100 \% / 100 \%$ & \\
\hline & T3: 6 & $75 \% / 75 \%$ & 0,013 \\
\hline & $\mathrm{T} 4: 5$ & $75 \% / 50 \%$ & \\
\hline \multirow[t]{2}{*}{$\mathrm{N}$} & N0: 24 & $92 \% / 81 \%$ & \\
\hline & $\mathrm{N}+: 4$ & $67 \% / 67 \%$ & 0,90 \\
\hline \multirow[t]{2}{*}{ M } & M0: 22 & $100 \% / 100 \%$ & \\
\hline & M1: 6 & $50 \% / 22 \%$ & 0,0056 \\
\hline \multirow[t]{4}{*}{ Estágio } & $\mathrm{I}: 8$ & $100 \% / 100 \%$ & \\
\hline & II: 11 & $86 \% / 64 \%$ & \\
\hline & III: 5 & $75 \% / 75 \%$ & \\
\hline & $\mathrm{IV}: 4$ & $100 \% / 100 \%$ & 0,61 \\
\hline \multirow[t]{2}{*}{ Cirurgia } & Total: 22 & $83 \% / 71 \%$ & \\
\hline & Parcial: 6 & $100 \% / 100 \%$ & 0,83 \\
\hline \multirow[t]{2}{*}{ Padrão Histológico } & MiI: 14 & $100 \% / 100 \%$ & \\
\hline & MaI: 14 & $67 \% / 44 \%$ & 0,02 \\
\hline
\end{tabular}


Tabela 4 - Relação da presença de metástases à distância com a idade, sexo, tamanho do nódulo, presença de metástase linfática e tipo de cirurgia nos casos de carcinoma das células de Hürthle.

\begin{tabular}{|c|c|c|c|c|c|c|c|c|c|c|c|c|}
\hline \multirow[t]{2}{*}{ M } & \multicolumn{2}{|c|}{ idade } & \multicolumn{4}{|c|}{$\mathbf{T}$} & \multicolumn{2}{|c|}{$\mathbf{N}$} & \multicolumn{2}{|c|}{ Cirurgia } & \multicolumn{2}{|c|}{ Sexo } \\
\hline & $\leq \mathbf{4 0}$ & $>40$ & 1 & 2 & 3 & 4 & No & $\mathrm{N}+$ & $<$ total & total & $\mathbf{M}$ & $\mathbf{F}$ \\
\hline M0 & 6 & 16 & 0 & 15 & 5 & 2 & 21 & 1 & 6 & 16 & 7 & 15 \\
\hline M1 & 2 & 4 & 0 & 2 & 1 & 3 & 3 & 3 & 0 & 6 & 1 & 5 \\
\hline$p$ & \multicolumn{2}{|c|}{0,82} & \multicolumn{4}{|c|}{0,07} & \multicolumn{2}{|c|}{$\mathbf{0 , 0 3}$} & \multicolumn{2}{|c|}{0,37} & \multicolumn{2}{|c|}{0,82} \\
\hline
\end{tabular}

A sensibilidade e especificidade da PAAF na detecção do $\mathrm{CCH}$ são de 78 e $18 \%$ respectivamente. Estes valores aumentam quando analisados no exame de congelação com 100 e $86 \%$ de sensibilidade e especificidade respectivamente ${ }^{7}$. É importante salientar que apenas dois $23 \%$ dos tumores foliculares apresentam critérios de malignidade, ao contrário dos 30 a $40 \%$ dos tumores com células de Hürthle que apresentam sinais de invasão ${ }^{7}$.

Na ausência de extensão extra-tireoidiana, metástases linfonodais ou à distância os critérios histológicos de malignidade são os padrões de invasão do $\mathrm{CCH}^{1}$. Os dois diferentes tipos de padrão histológico (minimamente ou maciçamente invasivos) podem ser usados como fatores prognósticos na evolução clínica de pacientes portadores de $\mathrm{CCH}^{1}$.

$\mathrm{Na}$ análise dos fatores prognósticos dos pacientes tratados no Instituto Nacional de Câncer (INCA), dados epidemiológicos como sexo e idade não estiveram relacionados com maior incidência de recidiva da doença ou a sobrevida dos pacientes. Na amostra deste estudo houve prevalência pelo sexo feminino, correspondendo a $71,4 \%$, valor semelhante ao resultado de $67,4 \%$ de prevalência do sexo feminino descrito por Dahl. Neste mesmo trabalho o autor descreveu que o sexo masculino apresentou incidência de malignidade em neoplasias de células de Hürthle de $67 \%$, resultado estatisticamente significante comparado a incidência de $36 \%$ de ma- lignidade no sexo feminino ${ }^{7}$.

Dahl também descreveu que os pacientes portadores de $\mathrm{CCH}$ apresentaram uma idade média e mediana aproximadamente 10 anos (58 e 61 anos respectivamente) ${ }^{7}$ maior que o grupo estudado em nosso estudo (50 e 51 anos respectivamente).

Stojadinovic analisou, dentre outros fatores, a influencia da idade e sexo com a sobrevida específica e o tempo livre de doença, não encontrando significância estatística nestas relações ${ }^{8}$. Em nosso estudo quando comparado a idade (menor ou igual a 40 anos X maior que 40 anos) e sexo com o tempo livre de doença e a sobrevida global não houve relação significante (Tabelas II e III). Ao contrário do que foi descrito por Bhattacharyya, que analisou 555 casos de carcinoma de $\mathrm{CCH}$ onde a idade mais avançada e o sexo masculino apresentaram diminuição da sobrevida ${ }^{9}$.

A extensão da ressecção da glândula tireóide em casos de neoplasias malignas bem diferenciadas ainda é motivo de controvérsia na literatura ${ }^{10-16}$. Essa discussão não é diferente nos casos de $\mathrm{CCH}$. Dos 28 pacientes analisados neste estudo, apenas seis $(21,4 \%)$ foram submetidos à ressecção cirúrgica menor que tireoidectomia total e em nenhum desses casos houve recidiva local, regional ou à distância. Não houve relação entre esta variável quando comparado à recidiva e sobrevida global ( $p=0,28$ e 0,83 respectivamente). Contudo

Tabela 5 - Relação do padrão histológico de invasão com a idade, sexo, tamanho do nódulo, presença de metástase linfática e à distância, estágio da doença e tipo de cirurgia nos casos de carcinoma das células de Hürthle.

\begin{tabular}{|c|c|c|c|c|c|c|c|c|c|c|}
\hline \multirow[t]{2}{*}{ Padrão Histológico } & \multicolumn{2}{|c|}{ Idade } & \multicolumn{4}{|c|}{$\mathbf{T}$} & \multicolumn{2}{|c|}{$\mathbf{N}$} & \multicolumn{2}{|c|}{$\mathbf{M}$} \\
\hline & $\leq \mathbf{4 0}$ & $>40$ & 1 & 2 & 3 & 4 & No & $\mathbf{N +}$ & M0 & M1 \\
\hline MiI & 3 & 11 & 0 & 10 & 4 & 0 & 14 & 0 & 14 & 0 \\
\hline MaI & 5 & 9 & 0 & 7 & 2 & 5 & 10 & 4 & 8 & 6 \\
\hline$p$ & \multicolumn{2}{|c|}{0,33} & \multicolumn{4}{|c|}{0,05} & \multicolumn{2}{|c|}{0,10} & \multicolumn{2}{|c|}{0,02} \\
\hline
\end{tabular}

\begin{tabular}{lllllrrrr}
\hline Padrão Histológico & \multicolumn{3}{c}{} & E & \multicolumn{3}{c}{ Cirurgia } & \multicolumn{2}{c}{ Sexo } \\
\cline { 2 - 10 } & I & II & III & IV & < total & total & M & F \\
\hline MiI & 5 & 7 & 2 & 0 & 5 & 9 & 2 & 12 \\
MaI & 3 & 4 & 3 & 4 & 1 & 13 & 6 & 8 \\
\hline $\boldsymbol{p}$ & & & 0,14 & & & 0,16 & & 0,20 \\
\hline
\end{tabular}


este dado deve ser considerado com cuidado, já que os pacientes que apresentam tumores mais avançados e extravasados são submetidos à tireoidectomia total. Stojadinovic em 2002, concluiu que não houve diferença entre a extensão da cirurgia quando relacionados com a sobrevida livre de doença e sobrevida específica da doença ${ }^{8}$.

O tamanho do tumor e a presença de metástase linfática não apresentaram significância quando relacionado com a incidência de recidiva. Já quando comparados com a sobrevida específica o tamanho do tumor apresentou significância estatística $(p=0,013)$ (Tabela II e III). Bhattacharyya descreveu que o tamanho do tumor teve relação direta com a diminuição da sobrevida ${ }^{9}$ e Stojadinovic, relacionou a pior sobrevida em pacientes com carcinoma de células de Hürthle que apresentavam metástases linfáticas ${ }^{1}$.

Dos 28 pacientes estudados com $\mathrm{CCH}$, apenas quatro $(14,2 \%)$ apresentaram metástase linfática. Três (75\%) destes pacientes apresentaram metástase à distância, sendo a relação entre estas variáveis estatisticamente significantes $(p=0,004)$.

As metástases à distância ocorreram em seis $(21,4 \%)$ pacientes. Em cinco $(83,4 \%)$ metástase óssea e em apenas um $(16,6 \%)$ caso ocorreu metástase pulmonar. Evans em 1998 também descreveu os ossos e os pulmões como os sítios mais freqüentes de metástases à distância em $\mathrm{CCH}$ e carcinomas foliculares ${ }^{17}$.

As variáveis que apresentaram significância estatística quando comparadas a sobrevida específica foram a presença de metástases à distância $(p=0,005)$, o tamanho do tumor $(p=0,013)$, e o padrão histológico de invasão $(p=0,02)$.
O padrão histológico de invasão também mostrou relação importante com a recidiva. Stojadinovic em 2001 descreveu que o padrão histológico de invasão maciça esteve relacionado com a recidiva em $73 \%$ dos pacientes, metástases linfáticas em $64 \%$ e sobrevida específica em $55 \%$ dos casos ${ }^{1}$. Em nossa casuística todos os casos que apresentaram metástases à distância apresentavam padrão de invasão maciça ao exame histopatológico (Tabela III), este mesmo padrão apresentou relação significante na análise univariada com a recidiva e sobrevida específica com o valor de $p$ igual a 0,02 e 0,002 respectivamente (Tabela III).

Os resultados deste trabalho não mostraram diferença na sobrevida entre os pacientes que se submeteram às operações parciais ou totais, estando estas associadas ou não ao esvaziamento cervical (Tabela III) e entre os pacientes que complementaram ou não a terapêutica com iodo radioativo. Entretanto, a ocorrência de vieses por não se tratar de estudo prospectivo e randomizado impede a conclusão sobre o melhor tratamento.

\section{CONCLUSÃO}

A presença de metástase à distância e o padrão histológico de invasão apresentaram relação estatisticamente significativa com a sobrevida livre de doença e específica. $\mathrm{O}$ padrão histológico de invasão maciça (MaI) apresentou relação estatisticamente significante com a presença de metástases à distância $(p=0,02)$ e o tamanho do tumor $(p=0,05)$. Pacientes com metástases linfáticas apresentaram maior incidência de metástases à distância $(p=0,004)$.

\begin{abstract}
Objective: Analyze prognostic factors for disease free survival and specific survival in patients with Hurthle Cell Carcinoma. Methods: We analyzed, retrospectively, the files of 28 patients treated in the Service of Head and Neck Surgery of the Brazilian National Cancer Institute/INCA from January 1983 to December 2002. Data regarding demographics, clinical aspects, therapeutics and histopathology were collected and related to disease-free survival and disease-specific survival. Patients were classified according to the AJCC 2002 staging system. Data was analyzed by the Epi Info 2002 software. Survival was analyzed by the Kaplan-Meier actuarial method. The median follow-up time was 69 months (range from 10 to 230). Results: Advanced stages ( $p=.03)$, distant metastases $(p=.03)$ and, particularly, the pattern of capsular invasion (widely invasive) $(p=.0027)$ influenced negatively the prognosis in the disease-free survival. Analyzing the relationship between specific survival, the pattern of capsular invasion (widely invasive) ( $p=.02), T$ stage $(p=.013)$ and, particularly, the presence of distant metastases $(p=.0056)$ presented statistical significance. Five and 10 year disease-free survival was $72 \%$ and $55 \%$, respectively, and 5 and 10 year disease-specific survival was $87 \%$ and $77 \%$, respectively. Conclusion: The presence of distant metastases and widely invasive tumors were the most important negative prognostic factors associated with Hurthle Cell Carcinomas in our study.
\end{abstract}

Key words: Oxyphil cells; Thyroid gland; Carcinoma; Prognosis; Thyroid neoplasms; Adenoma, oxyphilic; Disease-free survival.

\section{REFERÊNCIAS}

1. Stojadinovic A, Ghossein RA, Hoos A, Urist MJ, Spiro RH, Shah JP, Brennan MF, Shaha AR, Singh B. Hürthle cell carcinoma: a critical histopathologic appraisal. J Clin Oncol. 2001; 19(10):2616-25.

2. Tyler DS, Shaha AR, Udelsman RA, Sherman SI, Thompson NW, Moley JF, Evans DB. Thyroid cancer: 1999 Update. Ann Surg Oncol. 2000; 7(5):376-98.
3. Sanz RJ, Cerpa CF, Domínguez Errázuriz M, Franco Silva C, Jadresic Vargas A, Pérez PG, Phillipi RV. Carcinoma de células de Hurthle del tiroides: experiencia en 41 años. Rev Med Chile. 1999; 127(7):821-6.

4. Ng SC, Lin JD, Huang BY, Chen CH, Hsueh C, Lee N, Yen TC. Diagnosis and management of 34 Hürthle cell tumors. Changgeng Yi Xue Za Zhi. 1999; 22(3):445-52.

5. Yutan E, Clarck OH. Hürthle cell carcinoma. Curr Treat Options Oncol. 2001; 2(4):331-5. 
6. Khafif A, Khafif RA, Attie JN. Hürthle cell carcinoma: a malignancy of low-grade potential. Head Neck. 1999; 21(6):506-11.

7. Dahl LD, Myssiorek D, Heller KS. Hürthle cell neoplasms of the thyroid. Laryngoscope. 2002; 112:2178-80.

8. Stojadinovic A, Hoos A, Ghossein RA, Urist MJ, Leung DHY, Spiro RH et al. Hürthle cell carcinoma: A 60-year experience. Ann Surg Oncol. 2002; 9(2):197-203.

9. Bhattacharyya N. Survival and prognosis in Hürthle cell carcinoma of the thyroid gland. Arch Otolaryngol Head Neck Surg. 2003; 129(2):207-10.

10. Grant CS, Hay ID, Gough IR, Bergstralh EJ, Goellner JR, McConahey WM. Local recurrence in papillary thyroid carcinoma: is extent of surgical resection important? Surgery. 1988; 104(6):954-62

11. Chow SM, Law SC, Mendenhall WM, Au SK, Chan PT, Leung TW, Tong CC, Wong IS, Lau WH. Papillary thyroid carcinoma: prognostic factors and the role of radioiodine and external radiotherapy. Int J Radiat Oncol Biol Phys. 2002; 52(3):784-95.

12. Hay ID, Grant CS, Taylor WF, McConahey WM. Ipsilateral lobectomy versus bilateral lobar resection in papillary thyroid carcinoma: a retrospective analysis of surgical outcome using a novel prognostic scoring system. Surgery. 1987; 102(6):1088-94.

13. Tsang RW, Brierley JD, Simpson WJ, Panzarella T, Gospodarowicz MK, Sutcliffe SB. The effects of surgery, radioiodine, and external radiation therapy on the clinical outcome of patients with differentiated thyroid carcinoma. Cancer. 1998; 82 (2):375-88.
14. Hay ID, Grant CS, van Heerden JA, Goellner JR, Ebersold JR, Bergstralh EJ. Papillary thyroid microcarcinoma: a study of 535 cases observed in a 50-year period. Surgery. 1992; 112(6):113946; discussion 1146-7.

15. Tollefson HR, Shah JP, Huvos AG. Papillary carcinoma of the thyroid. Recurrence in the thyroid gland after initial surgical management. Am J Surg. 1972; 124(4):468-72.

16. Shah JP, Lorre TR, Dharker D, Strong EW. Lobectomy versus total thyroidectomy for differentiated carcinoma of the thyroid: a matched pair analysis. Am J Surg. 1993; 166(4):331-5.

17. Evans HL, Vassilopoulou-Sellin R. Follicular and Hurthle cell carcinomas of the thyroid: a comparative study. Am J Surg Pathol. 1998; 22(12):1512-20.

Como citar este artigo:

Manfro G, Dias FL, Lima RA, Barbosa MM, Soares Netto JR, Nascimento MF. Fatores prognósticos em pacientes com carcinoma de células de Hürthle. Rev Col Bras Cir. [serial on the Internet] 2006 Mar-Apr; 33(2). Available from URL: http://www.scielo.br/rcbc.

Endereço para correspondência:

Gabriel Manfro

Seção de Cirurgia de Cabeça e Pescoço - Instituto Nacional de Câncer Praça da Cruz Vermelha, $\mathrm{N}^{\circ} 23,6^{\circ}$ andar

20230-130 - Rio de Janeiro - RJ

E-mail: fdias@inca.gov.br 